\title{
Trastornos de la conducta alimentaria en adolescentes. Una mirada integral
}

\author{
Eating disorders in adolescents. A comprehensive approach \\ Verónica Gaete P. ${ }^{\mathrm{a}, \mathrm{b}}$, Carolina López C. ${ }^{\mathrm{a}, \mathrm{b}}$
}

aCentro SERJOVEN, Departamento de Pediatría y Cirugía Infantil Oriente, Facultad de Medicina, Universidad de Chile

bUnidad de Trastornos Alimentarios, Centro de Adolescentes y Jóvenes, Departamento de Pediatría, Clínica Las Condes

Recibido: 29 de noviembre de 2019, Aceptado: 21 de abril de 2020

¿Qué se sabe del tema que trata este estudio?

Los TCA son enfermedades muy serias que se inician habitualmente en la adolescencia y en general han aumentado en los países en desarrollo. Existe escasez de capacitación de los profesionales de la salud en este tema a nivel del país.
¿Qué aporta este estudio a lo ya conocido?

Una revisión actualizada sobre la epidemiología, etiología, el impacto en la salud integral, la presentación clínica y el tratamiento de los TCA más frecuentes en la adolescencia.

\section{Resumen}

Los trastornos de la conducta alimentaria (TCA) son enfermedades muy serias que se inician habitualmente en la adolescencia y en general han ido en aumento en los países en desarrollo. Actualmente, diversos factores obstaculizan su prevención, pesquisa y tratamiento en el país, siendo uno importante la escasez de capacitación de los profesionales de salud en este tema emergente. El objetivo de este artículo es contribuir al conocimiento que poseen los pediatras sobre los TCA en la adolescencia, mediante una revisión actualizada de la literatura en el tema. En esta publicación se abordan la principal clasificación internacional de los TCA en uso en la literatura científica actual y la epidemiología, etiología, el impacto en la salud integral, la presentación clínica y el tratamiento de los TCA más frecuentes en la adolescencia.

\section{Abstract}

Eating disorders (ED) are very serious diseases that usually begin in adolescence and have, in general, been increasing in developing countries. In the country, there are currently several factors that hinder their prevention, detection, and treatment, where the lack of training of health
Palabras clave:

Trastornos

alimentarios;

adolescente;

pediatría;

revisión de los

trastornos alimentarios

Keywords:

Eating disorders;

adolescent;

pediatrics;

eating disorders review 
professionals in this emerging issue appears as an important one. The purpose of this article is to contribute to the knowledge that pediatricians have about ED in adolescence, through an updated review of the literature on the subject. This publication addresses the main international classification of ED in use in the current scientific literature and the epidemiology, etiology, impact on comprehensive health, clinical presentation, and treatment of the most common ED in adolescence.

\section{Introducción}

Los TCA, descritos en la última versión del Manual Diagnóstico y Estadístico de Enfermedades Mentales (DSM-5) ${ }^{1}$ como Trastornos Alimentarios y de la Ingestión de Alimentos, son enfermedades muy serias, asociadas a una significativa morbilidad y mortalidad ${ }^{2,3}$, que en general han ido en aumento en los países en desarrollo ${ }^{4}$.

Diversos factores obstaculizan la prevención, pesquisa y el tratamiento de estas enfermedades actualmente en el país. Algunos son propios de los TCA -como la tendencia de quienes los sufren a negarlos o esconderlos 5 -, y otros dicen relación con la falta de preparación de nuestro sistema de salud para enfrentarlos adecuadamente, en especial, por la escasez de capacitación de los profesionales de salud en este tema emergente y de servicios adecuados para el manejo de los $\operatorname{casos}^{5,6}$.

Debido a lo anterior y a que se trata de enfermedades que se inician habitualmente en la adolescencia ${ }^{7}$, resulta esencial que los pediatras se familiaricen con ellas. Esta publicación tiene como objetivo contribuir al conocimiento que poseen los pediatras sobre los TCA en la adolescencia, mediante una revisión actualizada de la literatura en el tema.

\section{Tipos de trastornos alimentarios}

Las principales clasificaciones internacionales de los TCA corresponden al DSM-5 y la Undécima Edición de la Clasificación Estadística Internacional de Enfermedades (CIE-11) ${ }^{8}$. La primera, que es la más empleada en la literatura científica sobre el tema, los denomina Trastornos Alimentarios y de la Ingestión de Alimentos e incluye aquellos que muestra la tabla 1 .

Este artículo aborda los TCA más frecuentes en la adolescencia -Anorexia Nerviosa (AN), Bulimia Nerviosa (BN), Trastorno por Atracón (TA) y Otros Trastornos Alimentarios y de la Ingestión de Alimentos Especificados-, cuyos criterios diagnósticos se presentan en la tabla 2 de manera simplificada.

\section{Epidemiología}

Históricamente percibidos como patologías propias de países occidentales desarrollados, con el paso de los años los TCA se han detectado en diversas culturas y países de menor desarrollo, en los que en general han ido en aumento ${ }^{4}$. Asimismo, el perfil de quienes los presentan se ha ido ampliando, incluyendo hoy en día no solo a mujeres adolescentes de clase alta y excelente rendimiento académico, sino también a varones, personas pertenecientes a minorías, e individuos de diverso nivel socioeconómico, etnia y estado nutricional, que los inician a edades cada vez más tempranas ${ }^{2,9,10}$.

A nivel internacional, la prevalencia reportada en adolescentes previa al DSM-5, oscilaba entre 0.5\% y $1 \%$ para $\mathrm{AN}$ y $1-2 \%$ y $4 \%$ para $\mathrm{BN}^{9,11,12}$, llegando hasta $14 \%$ en el caso de los TCA No Especificados ${ }^{11}$. Los cambios en los criterios diagnósticos introducidos por el DSM-5 se tradujeron en un incremento de casos catalogados como AN y BN, y una reducción significativa de aquellos identificados como TCA No Especificados ${ }^{13}$. Por otra parte, la prevalencia de TA oscilaría entre 1 y $3 \%$ en adolescentes y niños ${ }^{14}$, siendo significativamente mayor entre aquellos que buscan tratamiento por obesidad ${ }^{15}$.

Respecto a la prevalencia de TCA entre adolescentes en Chile, Vicente y cols. encontraron 0,3\% en el último año en jóvenes de ambos sexos de 12 a 18 años, en una muestra nacional representativa ${ }^{16}$. La cifra contrasta con aquella evidenciada por Granillo y cols. ${ }^{17}$, quienes hallaron una prevalencia de vida de TCA (AN y $\mathrm{BN}$ ) de $3,8 \%$, en una muestra de la comunidad de adolescentes de sexo femenino (edad $\mathrm{x}=14$ años), de nivel socioeconómico medio a bajo, en Santiago. Los estudios sobre la prevalencia del riesgo de tener o desarrollar estos trastornos en este grupo etario, han mostrado cifras significativas en población escolarizada, que oscilan entre $7,4 \%$ y $12 \%$ y son mayores entre las mujeres $(8,3 \% \text { a } 23 \%)^{18-20}$.

\section{Etiología}

Se carece de una comprensión clara y completa de la causa de los TCA. La investigación a la fecha ha 
Tabla 1. Trastornos alimentarios y de la ingestión de alimentos (DSM-5)

- Pica

- Trastorno de rumiación

- Trastorno de evitación/restricción de la ingestión de alimentos

- Anorexia nerviosa

- Bulimia nerviosa

- Trastorno por atracón

- Otros trastornos alimentarios y de la ingestión de alimentos especificados (AN atípica, BN de frecuencia baja y/o duración limitada, TA de frecuencia baja y/o duración limitada, trastorno por purgas y síndrome de ingestión nocturna de alimentos)

- Trastornos alimentarios y de la ingestión de alimentos no especificados

American Psychiatric Association, $2013^{1}$.

evidenciado diversos factores biológicos, psicológicos, conductuales y socioambientales que interactuarían de manera compleja, contribuyendo a su aparición y mantención ${ }^{21,22}$.

Dentro de los biológicos se encuentran algunos genéticos, fisiológicos y neurobiológicos. Se ha demostrado una fuerte contribución genética en $\mathrm{AN}, \mathrm{BN}$ y TA, sin haberse identificado aún las variantes genéticas asociadas $^{22}$. La pubertad y la malnutrición por exceso constituyen ejemplos de factores fisiológicos asociados al desarrollo de TCA, y la desregulación en los sistemas dopaminérgico y serotoninérgico, más las alteraciones en los circuitos neurales relacionados con la recompensa y el autocontrol, ejemplos de factores neurobiológicos relacionados con estas enfermedades ${ }^{21,22}$. Los últimos años se ha comenzado a estudiar el rol que podría tener la microbiota intestinal en la etiopatogenia y curso de los TCA, debido a su influencia en diversos factores relevantes en estos trastornos, como la adiposidad, el metabolismo, el peso y algunos síntomas psicológicos (por ej., ansiedad), con hallazgos interesantes pero aún preliminares ${ }^{23,24}$.

Respecto de los factores psicológicos, se han identificado una amplia gama como predictores del inicio y mantención de los TCA, tanto temperamentales y de personalidad, como cognitivos, conductuales y emocionales. Ejemplos de ellos son el perfeccionismo, la urgencia negativa (reacción precipitada ante la angustia), la autoestima baja, el afecto negativo (depresión, ansiedad y/o culpa), el deterioro en el funcionamiento interpersonal, la insatisfacción corporal, y la preocupación excesiva por la alimentación, el peso y la figu$\mathrm{ra}^{21,25}$.

Así también, algunas conductas alimentarias se asocian al riesgo de desarrollar un TCA, como el hacer dieta, factor relevante como precipitante de TCA, en especial si se trata de dietas severas ${ }^{25,26}$.

En cuanto a los factores de riesgo socioambientales, existe una variedad de ellos, tanto de índole proximal como distal, que jugaría un rol en el inicio y la manten-

\section{Tabla 2. Criterios diagnósticos de los TCA más frecuentes en la adolescencia}

Anorexia nerviosa (AN)

Restricción de la ingesta de energía en relación a los requerimientos del individuo, llevando a un peso corporal significativamente bajo en el contexto de la edad, sexo, trayectoria del desarrollo y estado de salud. La distorsión de la imagen corporal, el miedo intenso a ganar peso, la falta de reconocimiento de la gravedad de la enfermedad y/o comportamientos que interfieren con la ganancia de peso, también están presentes

Bulimia nerviosa (BN)

Atracones (comer una gran cantidad de comida en un tiempo relativamente corto, con la sensación de pérdida de control) con comportamientos purgativos/compensatorios (por ej., vómito autoinducido, abuso de laxantes y/o diuréticos, mal uso de insulina, ejercicio excesivo, medicamentos para bajar de peso) una vez o más por semana, por al menos 3 meses. También pueden estar presentes la distorsión de la imagen corporal, un miedo intenso a ganar peso y la falta de reconocimiento de la gravedad de la enfermedad

Trastorno por atracón (TA)

Atracones, en ausencia de comportamientos compensatorios, al menos una vez por semana durante 3 meses. Los episodios de atracones se asocian con comer rápidamente, sin hambre, hasta sentirse incómodamente lleno, y/o con depresión, vergüenza o culpa

Otros trastornos alimentarios o de la ingestión de alimentos especificados

TCA que no cumple con todos los criterios de alguna de las categorías anteriores, pero tiene comportamientos específicos, tales como restricción de la ingesta, purgas y/o atracones, como características clave.

Estos comprenden:

1. AN Atípica

2. BN (de frecuencia baja y/o duración limitada)

3. TA (de frecuencia baja y/o duración limitada)

4. Trastorno purgativo

5. Síndrome de ingestión nocturna de alimentos

Adaptado de AED, $2016^{2}$. 
ción de los TCA. Ejemplos de los primeros son los comentarios críticos sobre la apariencia y la alimentación por parte de los padres, y los conflictos familiares no resueltos. Dentro de los segundos se encuentran, entre otros, el haber sido objeto de burlas (de diverso tipo, incluyendo aquellas por el peso y la figura), además de la presión percibida para ser delgado (para lograr el "ideal delgado", es decir, un cuerpo flaco con poca grasa corporal), la internalización de este "ideal delgado" y las expectativas positivas asociadas a la delgadez propias de las culturas occidentalizadas, que aumentan el riesgo de patología alimentaria especialmente en mujeres adolescentes y adultas jóvenes ${ }^{21,27}$.

Se cree que los individuos con TCA tendrían una predisposición biológica o genética al desarrollo de estas patologías, que es activada por factores ambientales ${ }^{21}$, pero los mecanismos subyacentes a la interacción entre los factores de riesgo deben aún aclararse.

\section{Impacto en la salud}

Los TCA se asocian a significativa morbilidad, mortalidad y compromiso severo de la calidad de vida ${ }^{2,3,28}$. Las tasas brutas de mortalidad reportadas en AN varían entre 0 y $22,5 \%$, destacando que el pronóstico es significativamente más favorable en adolescentes ${ }^{3}$.

Los TCA pueden comprometer prácticamente todos los órganos y sistemas corporales, y afectar el crecimiento y desarrollo ${ }^{2,10}$. Las complicaciones médicas (tabla 3) son habitualmente consecuencia de la desnutrición, los atracones y las purgas ${ }^{9}$, y la mayoría mejora al regresar estas alteraciones. Sin embargo, algunas son potencialmente irreversibles o incluso pueden llevar a la muerte.

Dentro de aquellas que pueden ser irreversibles destacan el retardo de crecimiento, la disminución de la densidad mineral ósea (DMO) y la atrofia cerebral. El crecimiento puede verse comprometido en los adolescentes con AN que no han alcanzado su talla final ${ }^{29}$. El impacto en la estatura es mayor si la enfermedad precede al estirón puberal, pico de velocidad de crecimiento y cierre epifisiario ${ }^{30,31}$, y es directamente proporcional a la severidad y duración de la $\mathrm{AN}^{31}$. Con la rehabilitación nutricional puede existir un crecimiento compensatorio, pero la talla final podría verse afecta$\mathrm{da}^{29}$. La AN se asocia también a baja DMO y a deterioro de la estructura y fortaleza óseas, con aumento del riesgo de fracturas ${ }^{32}$. Siendo la adolescencia una etapa crítica para el logro del pico de masa esquelética, el desarrollo de esta enfermedad en este período aumenta significativamente el riesgo de compromiso persistente de la salud ósea. El grado de deterioro de la DMO está muy influido por la mayor duración de la amenorrea y una edad de menarquia más tardía ${ }^{32}$. Si bien la recu- peración nutricional y la reanudación de la menstruación se asocian con cierta mejoría de la DMO, pueden persistir déficits ${ }^{32}$. Asimismo, durante la fase aguda de la AN existe una significativa reducción global de las sustancias gris y blanca a nivel cerebral, más pronunciada en adolescentes que en adultos ${ }^{33}$. La implicancia de estas alteraciones está en estudio, destacando que, si bien revierten con la recuperación nutricional, aún existen dudas de si lo hacen por completo en los adolescentes ${ }^{33,34}$.

Dentro de las complicaciones médicas de los TCA que pueden llevar a la muerte, destacan las cardiovasculares. Hasta un $80 \%$ de los pacientes con AN evi-

Tabla 3. Complicaciones médicas de los TCA

\begin{tabular}{|c|c|}
\hline Generales & $\begin{array}{l}\text { - Deshidratación } \\
\text { - Hipokalemia } \\
\text { - Hipomagnesemia } \\
\text { - Hiponatremia } \\
\text { - Amenorrea/otras alteraciones menstruales } \\
\text { - DMO baja/osteoporosis }\end{array}$ \\
\hline $\begin{array}{l}\text { Relacionadas } \\
\text { a la restricción } \\
\text { calórica y pérdida } \\
\text { de peso }\end{array}$ & $\begin{array}{l}\text { - Incapacidad para mantener la temperatura cor- } \\
\text { - Atrofia miocárdica } \\
\text { - Aumento de dispersión del intervalo QT } \\
\text { - Arritmias } \\
\text { - Otras alteraciones electrocardiográficas } \\
\text { - Prolapso de la válvula mitral } \\
\text { - Derrame pericárdico } \\
\text { - Retardo del vaciamiento gástrico y alteración de } \\
\text { la motilidad gastrointestinal (distensión abdomi- } \\
\text { - nal/plenitud postprandial/constipación) } \\
\text { - Hipoglicemia } \\
\text { - Alteración exámenes de función hepática } \\
\text { - Anemia/leucopenia/trombocitopenia } \\
\text { - Síndrome del eutiroídeo enfermo } \\
\text { - Retardo de crecimiento } \\
\text { - Atrofia cerebral }\end{array}$ \\
\hline $\begin{array}{l}\text { Relacionadas a } \\
\text { los atracones }\end{array}$ & - Dilatación aguda y ruptura gástrica (rara) \\
\hline $\begin{array}{l}\text { Relacionadas a } \\
\text { los vómitos }\end{array}$ & $\begin{array}{l}\text { - Alcalosis metabólica hipoclorémica } \\
\text { - Esofagitis } \\
\text { - Reflujo gastroesofágico } \\
\text { - Erosiones dentales } \\
\text { - Síndrome de Mallory-Weiss } \\
\text { - Ruptura esofágica o gástrica (rara) } \\
\text { - Neumonía aspirativa (rara) }\end{array}$ \\
\hline $\begin{array}{l}\text { Relacionadas } \\
\text { al abuso de } \\
\text { laxantes }\end{array}$ & $\begin{array}{l}\text { - Acidosis metabólica hiperclorémica } \\
\text { - Hiperuricemia } \\
\text { - Hipocalcemia } \\
\text { - Retención hídrica post suspensión de laxantes }\end{array}$ \\
\hline
\end{tabular}

Adaptado de Rosen, $2010^{11}$. Complemento en base a Rome, $2012^{9}$. 
dencia alteraciones del sistema cardiovascular ${ }^{35}$, que incluyen aquellas estructurales (por ej., derrame pericárdico, atrofia miocárdica y prolapso de la válvula mitral), alteraciones de la repolarización y la conducción (por ej., prolongación y aumento de la dispersión del intervalo QT), cambios hemodinámicos (por ej., bradicardia sinusal, hipotensión y cambios ortostáti$\cos )$ y alteraciones vasculares periféricas ${ }^{35,36}$. Mientras algunas son leves y reversibles, otras se asocian a mortalidad (son responsables de un tercio de las muertes observadas en esta patología), haciéndose más significativas en la medida que existe mayor compromiso nutricional ${ }^{35}$.

Cabe destacar que aquellas complicaciones que son consecuencia de la desnutrición pueden también observarse en pacientes que no llegan a evidenciar ese estado nutricional por antropometría, pero que han experimentado una pérdida de peso significativa, como aquellos con AN Atípica ${ }^{37,38}$.

Entre los problemas psicológicos más frecuentes asociados a los TCA se encuentran el aislamiento social y las dificultades para fortalecer el autoconcepto, la autoestima, la autonomía y la capacidad de intimar, por lo que estas enfermedades pueden interferir en el logro de las tareas propias de la adolescencia ${ }^{39,40}$.

La comorbilidad psiquiátrica es también común en los TCA, especialmente los síntomas depresivos, los trastornos ansiosos y el trastorno obsesivo-compulsivo $^{10,41}$. Así también, se asocian a trastornos de la personalidad, abuso de sustancias y comportamientos autoagresivos $^{10,41}$. La conducta suicida contribuye de manera significativa a la mortalidad asociada ${ }^{3}$.

Asimismo, las relaciones familiares se afectan seriamente en los $\mathrm{TCA}^{42}$, destacando que los padres se ven sometidos al intenso y prolongado estrés que genera el cuidado de estos pacientes, con aumento de sus propias posibilidades de presentar serios problemas de salud mental ${ }^{43-45}$.

\section{Presentación clínica}

Los TCA comienzan habitualmente en la adolescencia ${ }^{7}$, aunque su identificación ha aumentado entre niños menores, a partir de los 5 años de edad ${ }^{46}$. La AN se inicia con mayor frecuencia en la adolescencia temprana a media ${ }^{7}$, siendo notable su aumento de incidencia entre mujeres de 15 a 19 años $^{7,47}$. La BN comienza habitualmente en la adolescencia tardía ${ }^{7}$. Respecto del Trastorno por Atracón (TA), un estudio evidenció su pico de aparición a los 13,9 años $^{48}$ y otro a los 18-20 años $^{49}$, debiéndose hacer presente la falta de inclusión de varones en el segundo, pues se trata del TCA más prevalente entre éstos ${ }^{50}$. La gran mayoría de los TCA se presentan en mujeres, sin embargo, en las últimas décadas ha aumentado su diagnóstico en hombres ${ }^{7}$. La proporción sexo femenino: masculino que muestran los diversos estudios varía entre $3: 1$ y 18:151.

La sintomatología de los TCA puede ser diversa, incluyendo:

Conductas restrictivas, como reducción de las porciones de comida ingerida, ayunos, evitación de cierto tipo de alimentos (en especial los que contienen carbohidratos y grasas), presencia de reglas estrictas relacionadas con la alimentación (por ej., comer solo en ciertos horarios), conductas ritualizadas en la compra, preparación y consumo de alimentos (por ej., adquirir sólo alimentos considerados "saludables" o sin sellos de advertencia), y evitación de las comidas habituales en el hogar y del comer en situaciones sociales. Tienden a disimularse refiriendo falta de apetito, sensación de "estar lleno", desagrado por ciertos alimentos, náuseas, dolor abdominal, o mediante la adopción de dietas que excluyen alimentos (por ej., conversión al vegetarianismo).

Atracones (ver definición en la tabla 2), los que deben sospecharse en jóvenes que aumentan progresivamente de peso a pesar de evidenciar una ingesta disminuida o normal durante las comidas habituales.

Conductas compensatorias, tanto purgativas (vómitos autoinducidos y uso indebido de laxantes, diuréticos y/u otros medicamentos), como no purgativas (restricción alimentaria y/o ejercicio). Las primeras tienden a esconderse (por culpa, vergüenza o para evitar suspenderlas) y pueden pasar desapercibidas durante años. Deben sospecharse, por ej., cuando existen visitas frecuentes al baño durante y después de las comidas.

Ejercicio excesivo, el que es intenso, de alta frecuencia o de naturaleza compulsiva, y puede asociarse a consecuencias físicas y sociales negativas, tales como interferencia en la recuperación nutricional, lesiones osteomusculares e inasistencia a actividades con amistades.

Otras conductas destinadas al control del peso, tales como consumo disminuido o aumentado de líquidos, masticar frecuentemente chicle sin azúcar, abuso de endulzantes, uso de diversas sustancias con el fin de suprimir el apetito o bajar de peso (cafeína, té, tabaco -fumar-, drogas ilegales y suplementos nutricionales), y abuso de otros suplementos, como barras y polvos de proteína, y creatina (esto último en varones activos en el gimnasio $)^{2,52-54}$.

Conductas de chequeo corporal, que incluyen pesarse frecuentemente, mirar, tocar o medir reiteradamente algunas partes del cuerpo (por ej., abdomen, muslos y protuberancias de huesos específicos), mirarse repetidamente al espejo, y comparar el cuerpo con el de otros. Están destinadas a monitorizar el peso y/o figura de manera obsesiva. Por el contrario, pueden 
existir conductas de evitación corporal, como rehusar pesarse o evadir los espejos.

Otros comportamientos relacionados con la alimentación, como, por ejemplo, contar calorías, esconder o botar comida para evitar ingerirla, mentir para no comer, preparar las propias comidas para controlar su composición, cocinar para los demás sin comer lo que se prepara, y esconder la baja de peso usando ropas sueltas.

Los síntomas psicológicos asociados al TCA, que comprenden irritabilidad, disminución del ánimo, tendencia al aislamiento, aumento de la ansiedad (especialmente a las horas de comida), autocrítica general, dificultad para concentrarse debido a preocupación por la alimentación, el peso y la figura, y conducta suicida $^{13,55}$.

Tras los anteriores se encuentra la llamada Psicopatología del TCA, consistente en preocupación excesiva por el peso y la figura, sobrevaluación de su importancia en la autoestima, miedo intenso a ganar peso (incluso estando con bajo peso), insatisfacción con la imagen corporal importante y persistente (con distorsión de ella en la AN y percepción de "estar gordo" a pesar del bajo peso), y minimización o negación de la severidad de los síntomas ${ }^{55,56}$. En la AN existe muy poca conciencia de problema y la posibilidad de ingresar a tratamiento, y tener que recuperar peso, constituye una amenaza para los pacientes. En la BN existe mayor predisposición al cambio debido a la vivencia negativa de parte de los síntomas (atracones, purgas y -cuando existe- aumento de peso) pero a la vez resistencias a dejar algunas conductas percibidas como útiles para el control del peso (por ej., la restricción alimentaria). Por ello, es común que los adolescentes con TCA tiendan a esconder o negar sus síntomas y se resistan a ser llevados a una evaluación.

Existen diversos síntomas médicos que pueden evidenciar los pacientes con TCA, los que son generados en su gran mayoría por la restricción de la ingesta, los déficits nutricionales, los atracones y las conductas compensatorias (tabla 4). Las alteraciones al examen físico que pueden estar presentes en los TCA se especifican en la misma tabla. Respecto del estado nutricional, la AN se asocia a bajo peso o desnutrición, en la BN suele existir eutrofia o sobrepeso, y en el TA se observa en general sobrepeso u obesidad ${ }^{53,57}$.

El diagnóstico de los TCA en los casos más precoces (escolares o adolescentes tempranos) puede verse

\section{Tabla 4. Síntomas médicos y hallazgos al examen físico en adolescentes con TCA}

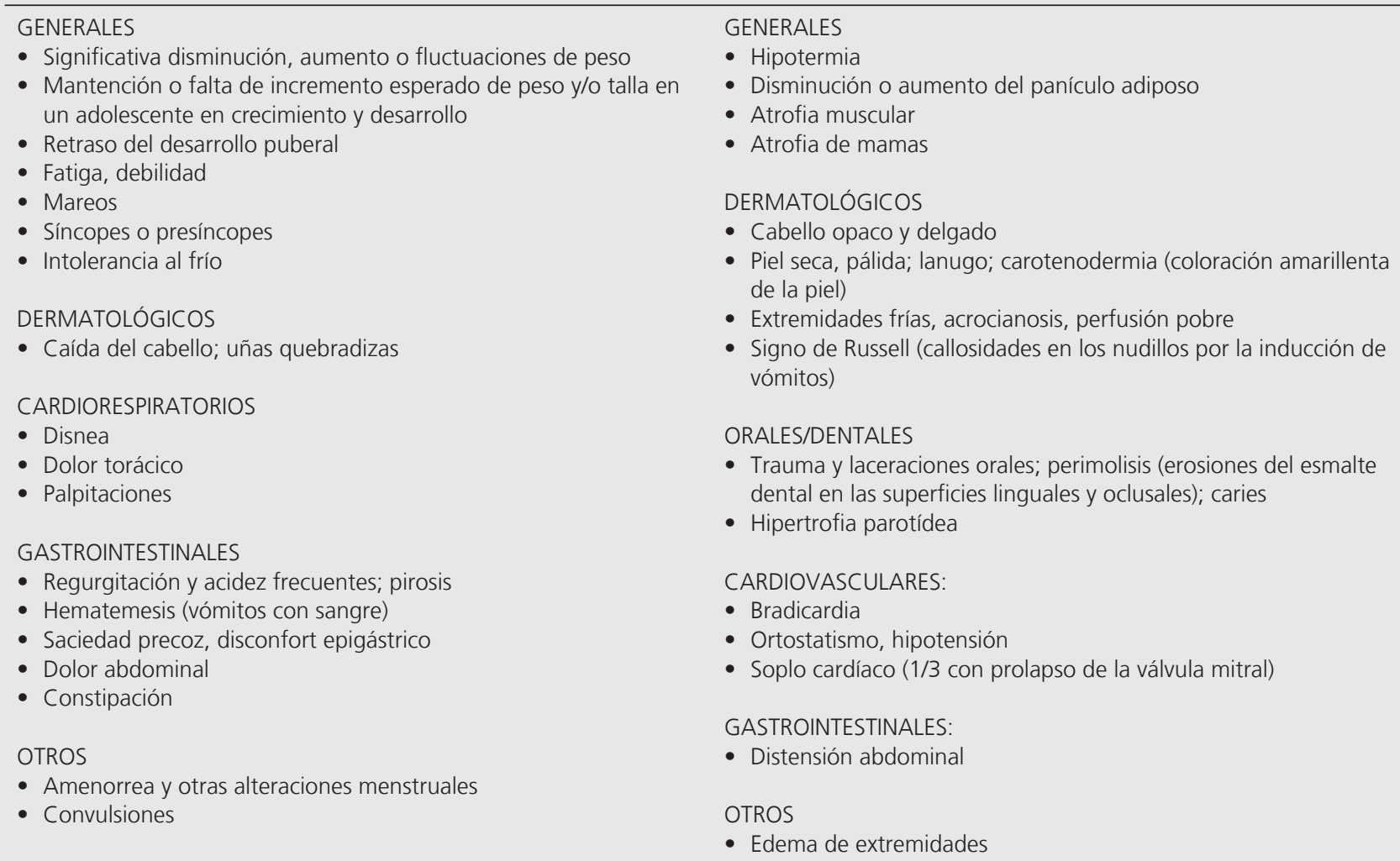

Adaptado de AED, 2016². Complemento en base a Rosen, $2010^{11}$ y Rome y Strandjord, $2016^{80}$ 
dificultado por limitaciones propias del desarrollo cognitivo y psicológico en estas etapas de la vida, cuando existe poca capacidad para reconocer y expresar sentimientos y pensamientos, $y$, por lo tanto, de interpretar y justificar las conductas que se presentan ${ }^{58}$. En estos casos, es importante considerar la presencia de conductas que indirectamente revelen los síntomas del TCA, en lugar de esperar que el joven los relate ${ }^{55}$. La presentación clínica evidencia también diferencias según sexo; los hombres presentan más deseo de aumentar su musculatura que de adelgazar -con excepción de aquellos con $\mathrm{AN}^{59}$-, y Otros Trastornos Alimentarios y de la Ingestión de Alimentos Especificados con mayor frecuencia que las mujeres ${ }^{60}$.

Cabe señalar, que a lo largo del tiempo estos pacientes transitan desde un tipo de TCA a otro con cierta frecuencia, siendo la migración desde una AN restrictiva hacia una AN de tipo con atracones/purgas o una BN, aquella más habitual ${ }^{61}$. Por otra parte, dentro del curso de los TCA son frecuentes las recaídas ${ }^{62} .31 \%$ de los pacientes con AN recae dentro de los dos años siguientes al alta del tratamiento ${ }^{63}$ y $21-55 \%$ de aquellos con BN lo hace en un plazo de 19 meses $^{64}$.

\section{Tratamiento}

Tanto el reconocimiento temprano, como el manejo adecuado de los TCA resultan cruciales para un pronóstico favorable ${ }^{50,65}$.

El tratamiento de estos adolescentes comprende manejo nutricional, psicológico, uso de psicofármacos para el TCA cuando está indicado, y abordaje de las complicaciones médicas y comorbilidades psiquiátri$\mathrm{cas}^{50,66}$

El manejo nutricional está dirigido a la corrección de las alteraciones nutricionales, la conducta alimentaria y otros comportamientos destinados a un control ponderal no saludable, además de al establecimiento de un manejo saludable y flexible de la alimentación y el peso ${ }^{67}$.

El tratamiento psicológico está destinado a apoyar la adherencia al tratamiento multidisciplinario e indicaciones nutricionales; aumentar la motivación a mantener un peso saludable y cesar las conductas propias del TCA; desarrollar una comprensión compartida de los problemas que subyacen al TCA; facilitar que el joven retome el desarrollo adolescente normal; y prevenir recaídas ${ }^{50,68}$. Diversas intervenciones psicológicas resultan útiles en estos casos, tales como la terapia conductual familiar (Family Based Therapy-FBT), Terapia Cognitivo-Conductual y su versión "mejorada", Terapia Interpersonal, Terapia Conductual-Dialéctica y Terapia Focalizada en la Adolescencia, varias de las cuales están respaldas por evidencia científica, aunque predominantemente en población adulta ${ }^{69-72}$. La FBT es la única que posee suficiente evidencia de efectividad en adolescentes con AN, por lo cual se plantea como tratamiento de primera línea en estos $\operatorname{casos}^{50,70}$.

El rol de los psicofármacos en el manejo de los TCA es limitado, con muy escasa evidencia en adolescentes, debiendo utilizarse principalmente para el tratamiento de la psicopatología comórbida ${ }^{73,74}$. En AN, se ha sugerido el uso de antipsicóticos con efectos sedativos, tales como la olanzapina, en casos en que el paciente se encuentra extremadamente agitado y con resistencia a la realimentación ${ }^{71}$. En adolescentes con BN, un estudio clínico demostró que la fluoxetina en dosis usadas en adultos (60 mg/día) añadida a la psicoterapia de apoyo, disminuyó significativamente los atracones y purgas ${ }^{75}$. Sin embargo, la evidencia es aún limitada y poco generalizable en este grupo etario, aunque su uso es común, dada la extrapolación de los resultados positivos obtenidos en adultos ${ }^{72,76}$. Prácticamente no hay estudios que evalúen la efectividad de los psicofármacos en adolescentes con TA. La lisdexanfetamina, aprobada para este trastorno en adultos, podría ser una opción promisoria ${ }^{77}$.

Otro elemento esencial en el tratamiento de los TCA en adolescentes es la inclusión de los padres, quienes deben formar parte activa y central en el manejo ${ }^{42,50,74,78}$.

Por otra parte, los pacientes con TCA deben ser regularmente monitoreados para detectar y manejar las complicaciones médicas asociadas a estas patologías y el síndrome de realimentación cuando corresponde. Asimismo, debe evaluarse y tratarse por la comorbilidad psiquiátrica que presenten ${ }^{2}$.

Existe consenso en que el tratamiento de los TCA en este grupo etario tiene que ser integral -dado el alcance biopsicosocial de estas patologías-, y realizado, de preferencia, por un equipo multidisciplinario especializado tanto en TCA como en adolescencia, por la complejidad de estas enfermedades y las particularidades de la etapa del desarrollo ${ }^{5}$. En forma idónea, tendría que contar con un pediatra especialista en adolescencia y profesionales del área de salud mental (psiquiatra y psicólogo) y de la nutrición (pediatra especialista en nutrición o nutricionista), más enfermeras y otros profesionales en los casos que requieran hospitalización ${ }^{5}$.

El tratamiento debiese comprender varios niveles de atención, articulados entre sí (ambulatorio, ambulatorio intensivo, hospitalización parcial/diurna, hospitalización completa), de modo de satisfacer las distintas necesidades que puede presentar el paciente ${ }^{79}$. La severidad de la enfermedad, su duración, la seguridad del paciente y las preferencias familiares determinarán el nivel necesario de atención ${ }^{10}$. Cabe destacar que el tratamiento ambulatorio es el de elección para los adolescentes y sólo debe recurrirse al manejo en otros ni- 
veles cuando no es factible (por falta de disponibilidad o riesgo del paciente) o a fracasado ${ }^{74}$.

\section{Conclusiones}

Los TCA son enfermedades muy serias que habitualmente se inician en la adolescencia y han ido en aumento en los países en desarrollo. Diversos factores obstaculizan su prevención, pesquisa y tratamiento actualmente en el país, siendo uno de ellos la escasez de capacitación de los profesionales de salud en este tema emergente, resultando esencial que los pediatras incrementen su nivel de conocimientos respecto de ellas.
Nota en relación con la terminología: En este artículo se utiliza el término "pediatra" para denominar a todos aquellos médicos que se dedican a la atención clínica de niños y adolescentes. Por otra parte, cuando se hace mención de "el" o "los" adolescente(s) o joven(es), se está aludiendo a personas de diferentes sexos de estos grupos etarios, y bajo la denominación "padre o padres" se incluyen a la(s) madre(s) y a otros adultos que ejerzan el rol parental.

\section{Conflicto de intereses}

Los autores declaran no tener conflicto de intereses.

\section{Referencias}

1. American Psychiatric Association. Diagnostic and statistical manual of mental disorders (5th ed.) DSM-5. Washington, DC: APA. 2013.

2. Academy for Eating Disorders (AED) report 2016. Eating disorders. A guide to medical care. Critical points for early recognition and medical risk management in the care of individuals with eating disorders. $3^{\text {rd }}$ Edition. 2016.

3. Jagielska G, Kacperska I. Outcome, comorbidity and prognosis in anorexia nervosa. Psychiatr Pol. 2017;51(2):205-18.

4. Kolar DR, Rodríguez DL, Chams MM, Hoek HW. Epidemiology of eating disorders in Latin America: a systematic review and meta-analysis. Curr Opin Psychiatry. 2016;29(6):363-71.

5. Gaete MV, López C, Matamala M. Trastornos de la conducta alimentaria en adolescentes y jóvenes. Parte I. Epidemiología, clasificación y evaluación inicial. Rev Med Clin Condes. 2012;23(5):566-78.

6. Vásquez N, Urrejola P, Vogel M. Actualizaciones en el manejo intrahospitalario de la anorexia nerviosa: recomendaciones prácticas. Rev Med Chile. 2017;145:650-6.

7. Golden NH, Schneider M, Wood C, AAP Committee on Nutrition. Preventing obesity and eating disorders in adolescents. Pediatrics. 2016;138(3):e20161649.

8. Organización Mundial de la Salud. Clasificación Internacional de Enfermedades, Undécima Revisión (CIE-11), 2018 [último acceso 29 octubre 2019]. Disponible en: https://icd.who.int/ browse11/1-m/es

9. Rome E. Eating disorders in children and adolescents. Curr Probl Pediatr Adolesc Health Care. 2012;42:28-44.
10. Campbell K, Peebles R. Eating disorders in children and adolescents: state of the art review. Pediatrics. 2014;34:582-92.

11. Rosen D. Identification and management of eating disorders in children and adolescents. Pediatrics. 2010;126:1240-53.

12. López C, Treasure J. Trastornos de la conducta alimentaria en adolescentes: descripción y manejo. Rev Med Clin Condes. 2011;22(1):85-97.

13. Herpertz-Dahlmann B. Adolescent eating disorders: update on definitions, symptomatology, epidemiology, and comorbidity. Child Adolesc Psychiatr Clin N Am. 2015;24(1):177-96.

14. Bohon C. Binge eating disorder in children and adolescents. Child Adolesc Psychiatr Clin N Am. 2019;28(4):549-55.

15. Cebolla A, Perpiñá C, Lurbe E, AlvarezPitti J, Botella C. Prevalencia del trastorno por atracón en una muestra clínica de obesos. An Pediatr (Barc.). 2012;77(2):98102.

16. Vicente B, Saldivia S, De la Barra F, Melipillán R, Valdivia M, Kohn R. Salud mental infanto-juvenil en Chile y brechas de atención sanitarias. Rev Med Chile. 2012;140:447-57.

17. Granillo MT, Grogan-Kaylor A, Delva J, Castillo M. Eating disorders among a community-based sample of Chilean female adolescents. J Res Adolesc. 2011;21(4):762-8.

18. Correa M, Zubarew T, Silva P, Romero M. Prevalencia de riesgo de trastornos alimentarios en adolescentes mujeres escolares de la Región Metropolitana. Rev Chil Pediatr. 2006;77(2):153-60.

19. Behar R, Alviña M, González T, Rivera N. Detección de actitudes y/o conductas predisponentes a trastornos alimentarios en estudiantes de enseñanza media de tres colegios particulares de Viña del Mar. Rev Chil Nutr. 2007;34(3):240-9.

20. Urzúa A, Castro S, Lillo A, Leal C.
Prevalencia de riesgo de trastornos alimentarios en adolescentes escolarizados del norte de Chile. Rev Chil Nutr. 2011;38(2):128-35.

21. Bakalar JL, Shank LM, Vannucci A, Radin RM, Tanofsky-Kraff M. Recent advances in developmental and risk factor research on eating disorders. Curr Psychiatry Rep. 2015;17(6):42.

22. Schaumberg K, Welch E, Breithaupt L, et al. The science behind the Academy for Eating Disorders' nine truths about eating disorders. Eur Eat Disord Rev. 2017;25(6):432-50.

23. Carr J, Kleiman SC, Bulik CM, BulikSullivan EC, Carroll IM. Can attention to the intestinal microbiota improve understanding and treatment of anorexia nervosa? Expert Rev Gastroenterol Hepatol. 2016;10:565-9.

24. Schwensen HF, Kan C, Treasure J, Høiby $\mathrm{N}$, Sjögren M. A systematic review of studies on the faecal microbiota in anorexia nervosa: future research may need to include microbiota from the small intestine. Eat Weight Disord. 2018;23:399418.

25. Stice E, Gau JM, Rohde P, Shaw H. Risk factors that predict future onset of each DSM-5 eating disorder: Predictive specificity in high-risk adolescent females. J Abnorm Psychol. 2017;126(1):38-51.

26. Patton GC, Selzer R, Coffey C, Carlin JB, Wolfe R. Onset of adolescent eating disorders: population based cohort study over 3 years. BMJ. 1999;318(7186):765-8.

27. Culbert KM, Racine SE, Klump KL. Research review: what we have learned about the causes of eating disorders - a synthesis of sociocultural, psychological, and biological research. J Child Psychol Psychiatry. 2015;56(11):1141-64.

28. Winkler LA, Christiansen E, Lichtenstein MB, Hansen NB, Bilenberg N, Støving RK. Quality of life in eating disorders: 
a meta-analysis. Psychiatry Res. 2014;219(1):1-9.

29. Peebles R, Sieke EH. Medical complications of eating disorders in youth. Child Adolesc Psychiatr Clin N Am. 2019;28(4):593-615.

30. Katzman D. Medical complications in adolescents with anorexia nervosa: a review of the literature. Int J Eat Disord 2005;37(suppl):S52-S9.

31. Misra M. Long-term skeletal effects of eating disorders with onset in adolescence. Ann NY Acad Sci. 2008;1135:212-8.

32. Misra M, Golden NH, Katzman DK. State of the art systematic review of bone disease in anorexia nervosa. Int J Eat Disord. 2016;49(3):276-92.

33. Seitz J, Konrad K, Herpertz-Dahlmann B. Extend, pathomechanism and clinical consequences of brain volume changes in anorexia nervosa. Curr Neuropharmacol. 2018;16(8):1164-73.

34. Seitz J, Bühren K, von Polier GG, Heussen N, Herpertz-Dahlmann B, Konrad K. Morphological changes in the brain of acutely ill and weight-recovered patients with anorexia nervosa. A meta-analysis and qualitative review. Z Kinder Jugendpsychiatr Psychother. 2014;42(1):7-17.

35. Spaulding-Barclay MA, Stern J, Mehler PS. Cardiac changes in anorexia nervosa. Cardiol Young. 2016;26(4):623-8.

36. Sachs KV, Harnke B, Mehler PS, Krantz MJ. Cardiovascular complications of anorexia nervosa: a systematic review. Int J Eat Disord. 2016; 49(3):238-48.

37. Garber A, Cheng J, Accurso E, et al. Weight loss and illness severity in adolescents with atypical anorexia nervosa. Pediatrics. 2019;144(6):e20192339.

38. Whitelaw M, Lee KJ, Gilbertson H, Sawyer SM. Predictors of complications in anorexia nervosa and atypical anorexia nervosa: Degree of underweight or extent and recency of weight loss? J Adolesc Health. 2018;63(6):717-23.

39. Fitzpatrick KK, Moye A, Hoste R, Lock J, le Grange D. Adolescent focused psychotherapy for adolescentes with anorexia nervosa. J Contemp Psychother. 2009;40(1):31-9.

40. Treasure J, Schmidt U: Eating disorders and the concept of working with families and other carers. En: Treasure J, Schmidt U, Macdonald P, eds. The clinician's guide to collaborative caring in eating disorders; East Sussex, UK: Routledge, 2010:7-19.

41. Herpertz-Dahlmann B, Holtkamp K, Konrad K. Eating disorders: anorexia and bulimia nervosa. En: Aminoff MJ, Boller F, Swaab DF. Handbook of clinical neurology, vol. 106 (3rd series). Schlaepfer TE, Nemeroff CB, eds.
Neurobiology of psychiatric disorders; Amsterdam, Holanda: Elsevier BV, 2012:447-62.

42. Treasure J, Schmidt U. The cognitiveinterpersonal maintenance model of anorexia nervosa revisited: a summary of the evidence for cognitive, socioemotional and interpersonal predisposing and perpetuating factors. J Eat Disord. 2013;1:13.

43. Kyriacou O, Treasure J, Schmidt U. Understanding how parents cope with living with someone with anorexia nervosa: modelling the factors that are associated with carer distress. Int J Eat Disord. 2008;41(3):233-42.

44. Zabala MJ, Macdonald P, Treasure J. Appraisal of caregiving burden, expressed emotion and psychological distress in families of people with eating disorders: a systematic review. Eur Eat Disord Rev. 2009;17(5):338-49.

45. Sepulveda AR, Graell M, Berbel E, et al. Factors associated with emotional well-being in primary and secondary caregivers of patients with eating disorders. Eur Eat Disord Rev. 2012;20(1):e78-e84.

46. Smink F, Van Hoeken D, Hoek HW. Epidemiology of eating disorders: Incidence, prevalence and mortality rates. Curr Psychiatry Rep. 2012;14(4):406-14.

47. Micali N, Hagberg KW, Petersen I, Treasure, JL. The incidence of eating disorders in the UK in 2000-2009: findings from the General Practice Research Database. BMJ Open. 2013;3(5):e002646.

48. Smink F, Van Hoeken D, Oldehinkel AJ, Hoek HW. Prevalence and severity of DSM-5 eating disorders in a community cohort of adolescents. Int J Eat Disord. 2014;47(6):610-9.

49. Stice E, Marti CN, Rohde P. Prevalence, incidence, impairment, and course of the proposed DSM- 5 eating disorder diagnoses in an 8-year prospective community study of young women. J Abnorm Psychol. 2013;122(2):445-57.

50. National Institute for Health and Care Excellence. Eating disorders: recognition and treatment. Full guideline. Version 2.0. 2017 [último acceso 26 de febrero de 2019]. Disponible en: https://www.nice. org.uk/guidance/ng69

51. Raevuori A, Keski-Rahkonen A, Hoek HW. A review of eating disorders in males. Curr Opin Psychiatry. 2014;27(6):426-30.

52. Gómez-Candela C, Palma S, Mijánde-la-Torre A, et al. Consenso sobre la evaluación y el tratamiento nutricional de los trastornos de la conducta alimentaria: anorexia nerviosa. Nutr Hosp. 2018;35(1):11-48.

53. Gómez-Candela C, Palma S, Miján-
de-la-Torre A, et al. Consenso sobre la evaluación y el tratamiento nutricional de los trastornos de la conducta alimentaria: bulimia nerviosa, trastorno por atracón y otros. Nutr Hosp. 2018;35(1):49-97.

54. Achiro R, Theodore P. Excessive workout supplement use: an emerging eating disorder in men? Paper session, $123^{\text {rd }}$ American Psychologycal Association Meeting. Toronto, Canadá, 2015.

55. Bryant-Waugh R, Lask B: Overview of eating disorders in childhood and adolescence. En: Lask B, Bryant-Waugh $\mathrm{R}$, eds. Eating disorders in childhood and adolescence. $4^{\mathrm{a}}$ edición; Londres, Reino Unido: Routledge, 2013:33-49.

56. Fairburn C. Cognitive behavior therapy and eating disorders; New York, USA: Guilford, 2008.

57. Bulik CM, Marcus MD, Zerwas S, Levine MD, La Via M. The changing "weightscape" of bulimia nervosa. Am J Psychiatry. 2012;169(10):1031-6.

58. Gordon KH, Holm-Denoma JM, Douglas VJ, Crosby R, Wonderlich SA: The classification of eating disorders. En: Agras WS, Robinson A, eds. The Oxford handbook of eating disorders. 2nd ed.; New York, USA: Oxford University Press, 2018:9-23.

59. Limbers CA, Cohen LA, Gray BA. Eating disorders in adolescent and young adult males: prevalence, diagnosis, and treatment strategies. Adolesc Health Med Ther. 2018;9:111-6.

60. Kinasz K, Accurso EC, Kass AE, Le Grange D. Does sex matter in the clinical presentation of eating disorders in youth? J Adolesc Health. 2016;58(4):410-6.

61. Eddy KT, Dorer DJ, Franko DL, Tahilani K, Thompson-Brenner H, Herzog DB. Diagnostic crossover in anorexia nervosa and bulimia nervosa: implications for DSM-V. Am J Psychiatry. 2008;165(2):245-50.

62. Herpertz-Dahlmann B. Treatment of eating disorders in child and adolescent psychiatry. Curr Opin Psychiatry. 2017;30(6):438-45.

63. Berends, T Boonstra N, van Elburg A. Relapse in anorexia nervosa: a systematic review and meta-analysis. Curr Opin Psychiatry. 2018;31(6):445-55.

64. Olmsted MP, Kaplan AS, Rockert W. Defining remission and relapse in bulimia nervosa. Int J Eat Disord. 2005;38(1):1-6.

65. Treasure J, Russell G. The case for early intervention in anorexia nervosa: theoretical exploration of maintaining factors. Br J Psychiatry. 2011;199(1):5-7.

66. Gowers S, Green L: Eating disorders and their management. En: Gowers S, Green L. Eating disorders: cognitive behaviour therapy with children and young people; London, UK: Routledge, 2009:3-18.

67. Herrin M: Basic nutrition counseling. 
En: Herrin M. Nutrition counseling in the treatment of eating disorders; Nueva York, EE. UU.: Brunner-Routledge, 2003:3-24.

68. Lask B, Bryant-Waugh R. Individual psychoterapy. En: Lask B, Bryant-Waugh R (eds.) Eating disorders in childhood and adolescence; New York; USA: Routledge, 2013:281-300.

69. Lock J. Treatment of adolescent eating disorders: progress and challenges. Minerva Psichiatr. 2010;51(3):207-16.

70. Lock J. An update on evidence-based psychosocial treatments for eating disorders in children and adolescents. J Clin Child Adolesc Psychol. 2015;44(5):707-21.

71. Resmark G, Herpertz S, HerpertzDahlmann B, Zeeck A. Treatment of anorexia nervosa-new evidence-based guidelines. J Clin Med. 2019;8(2).pii:E153.
72. Hail L, Le Grange D. Bulimia nervosa in adolescents: prevalence and treatment challenges. Adolesc Health Med Ther. 2018;9:11-6.

73. Schmidt U, Adan R, Böhm I, et al. Eating disorders: the big issue. Lancet Psychiatry. 2016;3(4):313-5.

74. Lock J, La Via MC. American Academy of Child and Adolescent Psychiatry (AACAP) Committee on Quality Issues (CQI). Practice parameter for the assessment and treatment of children and adolescents with eating disorders. J Am Acad Child Adolesc Psychiatry. 2015;54(5):412-25.

75. Kotler LA, Devlin MJ, Davies M, Walsh BT. An open trial of fluoxetine for adolescents with bulimia nervosa. J Child Adolesc Psychopharmacol 2003;13(3):329-35.

76. van den Heuvel LL, Jordaan GP. The psychopharmacological management of eating disorders in children and adolescents. J Child Adolesc Ment Health. 2014;26(2):125-37.

77. Srivastava G, O’Hara V, Browne N. Use of lisdexanfetamine to treat obesity in an adolescent with severe obesity and binge eating. Children (Basel). 2019;4;6(2).

78. American Psychiatric Association. Practice guideline for the treatment of patients with eating disorders. Washington, DC.: APA. 2006.

79. Golden NH, Katzman DK, Kreipe RE, et al; Society for Adolescent Medicine. Eating disorders in adolescents: position paper of the Society for Adolescent Medicine. J Adolesc Health. 2003;33(6):496-503.

80. Rome ES, Strandjord SE. Eating disorders. Pediatr Rev. 2016;37(8):323-36. 